\title{
Perfil sociodemográfico, de saúde e hábitos de estilo de vida de idosos longevos de um município do interior da Bahia
}

\author{
Sociodemographic, health and lifestyle habits profile of long age elderly in a municipality in the \\ interior of Bahia \\ Perfil sociodemográfico, salud y hábitos de vida de ancianos longevos en un municipio del interior \\ de Bahia
}

Recebido: 10/02/2021 | Revisado: 16/02/2021 | Aceito: 10/03/2021 | Publicado: 18/03/2021

\author{
Narcisio Rios Oliveira \\ ORCID: https://orcid.org/0000-0001-6981-2988 \\ Centro Universitário Adventista de São Paulo, Brasil \\ E-mail: narcisiorios@gmail.com \\ Elias Ferreira Porto \\ ORCID: https://orcid.org/0000-0001-8326-2054 \\ Centro Universitário Adventista de São Paulo, Brasil \\ E-mail: eliasfporto@gmail.com
}

\begin{abstract}
Resumo
Objetivo: Caracterizar o perfil sociodemográfico, de saúde e hábitos de estilo de vida de idosos longevos de um munícipio do interior da Bahia. Método: Trata-se de um estudo transversal, descritivo de base populacional realizado com idosos entre 80 e 107 anos, localizados por meio da técnica "bola de neve", após aceite em participar da pesquisa os idosos foram submetidos a teste cognitivo e aqueles com estado cognitivo preservado responderam a um questionário elaborado pelos pesquisadores sobre o perfil sociodemográfico, condições de saúde e hábitos vida. Resultados: A amostra final deste estudo foi composta por 87 idosos longevos, maioria do sexo feminino 52,9\%, sem escolaridade 71,3\%, com renda familiar entre 1 e 3 salários-mínimos 73,6\%, portadores de hipertensão arterial sistêmica 46\%. Quanto aos hábitos de estilo de vida, os longevos pesquisados relataram não fazer uso de fumo 87,4\% e bebidas alcoólicas $85,1 \%$, possuir estado nutricional adequado $37,9 \%$, não praticar atividade física $60,9 \%$, viver em ambientes não estressantes $83,9 \%$, dormir $\geq 8$ horas de sono $92 \%$. Conclusão: Estes achados, podem contribuir para a melhor compreensão do perfil de longevos residentes de pequenas cidades com baixo IDH de forma que ações voltadas a promoção da saúde e hábitos de vida saudáveis sejam fortalecidos e implementados levando em consideração este público.
\end{abstract}

Palavras-chave: Estilo de vida; Saúde do idoso; Longevidade; Idoso de 80 anos ou mais.

\begin{abstract}
Objetive: Characterize the sociodemographic, health and lifestyle habits of long-lived elderly in a municipality in the interior of Bahia. Methodology: This is a cross-sectional, descriptive, population-based study carried out with elderly people between 80 and 107 years old, after accepting to participate in the research, the elderly were for submeted cognitive testing and those with preserved cognitive status answered a questionnaire prepared by researchers on the sociodemographic profile, health conditions and life habits. Results: The final sample of this study was composed of 87 long-lived elderly, the majority female $52.9 \%$, without schooling $71.3 \%$, with family income between US $\$ 194.0$ and US\$582.0 73.6\%, with systemic arterial hypertension $46 \%$. As for lifestyle habits, the oldest olds surveyed reported not using tobacco $87.4 \%$ and alcoholic beverages $85.1 \%$, having an adequate nutritional status $37.9 \%$, not practicing physical activity $60.9 \%$, living in environments non-stressful $83.9 \%$, sleep $\geq 8$ hours of sleep $92 \%$. Conclusion: These findings may contribute to a better understanding of the profile of long-lived residents of small cities with low HDI so that actions aimed at promoting health and healthy living habits are strengthened and implemented taking this audience into account.
\end{abstract}

Keywords: Life style; Health of the elderly; Longevity; Aged 80 and over.

\section{Resumen}

Objetivo: Caracterizar los hábitos sociodemográficos, de salud y de estilo de vida de los ancianos longevos de un municipio del interior de Bahía. Método: Se trata de un estudio poblacional, descriptivo, transversal, realizado con ancianos entre 80 y 107 años, ubicado mediante la técnica de la "bola de nieve", tras aceptar participar en la investigación, los ancianos fueron sometidos a una prueba cognitiva y aquellos con estado cognitivo conservado 
respondieron un cuestionario elaborado por los investigadores sobre el perfil sociodemográfico, condiciones de salud y hábitos de vida. Resultados: La muestra final de este estudio estuvo compuesta por 87 ancianos longevos, en su mayoría mujeres 52,9\%, sin escolaridad 71,3\%, con renta familiar entre 1 y 3 salarios mínimos 73,6\%, con hipertensión arterial sistémica 46\%. En cuanto a los hábitos de vida, los ancianos encuestados reportaron no consumir tabaco $87,4 \%$ y bebidas alcohólicas $85,1 \%$, tener un estado nutricional adecuado $37,9 \%$, no practicar actividad física $60,9 \%$, vivir en ambientes no estresantes $83,9 \%$, dormir $\geq 8$ horas de dormir $92 \%$. Conclusión: Estos hallazgos pueden contribuir a una mejor comprensión del perfil de los residentes longevos de pequeñas ciudades con bajo IDH para que las acciones dirigidas a promover la salud y hábitos de vida saludables se fortalezcan e implementen teniendo en cuenta este público.

Palabras clave: Estilo de vida; Salud del anciano; Longevidad; Anciano de 80 o más años.

\section{Introdução}

O Brasil está em um processo acelerado de envelhecimento demográfico, a população idosa é o segmento etário que mais cresce no país, apresentando taxas de mais de 4\% de crescimento ao ano no período de 2012 a 2020 (IBGE, 2015), os grupos populacionais mais velhos, e aqueles acima dos 80 anos em especial, estão crescendo proporcionalmente mais rápido do que qualquer outra faixa etária (ILC Brasil, 2015).

Ao classificar o envelhecimento segundo o critério demográfico por faixa etária, Minayou e Firmo (2019) classificam como "velho-jovem" ou também conhecida como "terceira idade" os idosos cuja faixa etária está entre 60 e 79 anos, enquanto que idosos com mais de 80 anos, são considerados como "velho-velho", também conhecidos como idosos da "quarta idade" e/ou longevos, ainda segundo os autores, um outro parâmetro utilizado para classificar o envelhecimento, agora de maneira individualizada é o que os distingue por meio da "herança genética, personalidade e forma de levar a vida".

Com o processo de envelhecimento, alterações associadas ao acúmulo de danos causados ao longo dos anos pela interação entre fatores genéticos e hábitos não saudáveis de estilo de vida, podem desencadear uma maior procura pelos serviços de saúde, como reflexo do desenvolvimento de doenças crônicas não transmissíveis (DCNT), além de outros aspectos relacionados a maior incapacidade, uso de medicamentos, dentre outros (Sardinha et al., 2020).

A partir do trabalho produzido por Dan Buettner no ano de 2005, o mundo despertou seus olhos para 5 áreas do globo onde existem um alto percentual demográfico de idosos centenários, ou seja, indivíduos com idade igual ou superior a 100 anos (Buettner \& Skemp, 2016). A partir de então, estas cinco regiões, a saber: Loma Linda (EUA), Nicoya (Costa Rica), Sardenha (Itália), Ikaria (Grécia) e Okinawa (Japão), passaram a ser conhecidas como Blue Zones® (Buettner \& Skemp, 2016; Govidaraju, Atzmon \& Barzilai, 2015).

Um fator de destaque para as Blue Zones® mencionadas por Buettner e Skemp (2016) está no fato de estarem localizadas em países cujo Índice de Desenvolvimento Humano (IDH) é considerado alto como o da Costa Rica, ou muito alto como é o caso dos EUA, Itália, Grécia e Japão (UNDP, 2015), o que corrobora com Govindaraju, Atzmon e Barzilai (2015), ao afirmarem que tais regiões podem ser mais propicias a uma vida útil mais longa.

Diante do exposto, torna-se de fundamental importância a necessidade da compreensão dos fatores sociodemográficos e de saúde da população idosa, em especial, a população longeva que dadas as suas características e fragilidades está mais suscetível ao desenvolvimento de DCNT o que pode comprometer significativamente o seu estilo de vida, saúde e longevidade, com destaque a cidade de Quixabeira, localizada na região semiárida do interior da Bahia (Lima, Reis \& Souza, 2005), por apresentar-se como uma região com alta proporção de centenários (IBGE, 2010b), mesmo estando localizada numa cidade cujo IDH, segundo o Programa das Nações Unidas para o Desenvolvimento - PNUD (2013) é considerado baixo.

Em 2010, segundo o último censo demográfico realizado pelo IBGE, o município de Quixabeira possuía 9.554 habitantes, destes 607 com idade igual ou superior a 70 anos, sendo 312 mulheres, das quais 100 eram octogenárias e 21 nonagenárias, 295 dos habitantes eram homens com idade igual ou superior a 70 anos, dos quais 83 eram octogenários, 23 
nonagenários e 3 centenários (IBGE, 2010).

Diante do exposto este estudo tem por objetivo caracterizar o perfil sociodemográfico, de saúde e hábitos de estilo de vida de idosos longevos do município de Quixabeira no estado da Bahia.

\section{Metodologia}

Trata-se de um estudo transversal, descritivo de base populacional com amostra de 87 idosos com idade igual ou superior a 80 anos, de ambos os sexos, do município de Quixabeira - Bahia. Os participantes foram convidados a participar do estudo por meio da técnica "bola de neve", tendo sido utilizada por se tratar de um método frequentemente usado na realização de pesquisas onde a população a ser estudada é rara ou desconhecida (Dewes, 2013), onde receberam orientações sobre a pesquisa e puderam manifestar o interesse assinando o Termo de Consentimento Livre e Esclarecido - TCLE, na presença de um familiar ou cuidador.

Este trabalho teve seu início após aprovação do Comitê de Ética em Pesquisa - CEP do Centro Universitário Adventista de São Paulo - UNASP, sob o parecer no3.785.915. A coleta de dados para a pesquisa aconteceu entre os meses de dezembro de 2019 e janeiro de 2020, onde os idosos foram convidados a participar da pesquisa e entrevistados em um único momento em seus respectivos domicílios. A fim de garantir a veracidade das informações prestadas os idosos foram inicialmente submetidos ao teste de avaliação do estado cognitivo "Mini-Exame do Estado Mental - MEEM", seguindo as recomendações e pontos de corte propostos pelo Ministério da Saúde (BRASIL, 2006).

Os idosos que após realização do MEEM apresentaram estado cognitivo preservado, foram convidados a responder um questionário sociodemográfico e de hábitos de estilo de vida elaborado pelos pesquisadores, este questionário incluiu informações relacionadas a caracterização individual (sexo, idade, raça, religião, escolaridade, estado civil, número de filhos, arranjo domiciliar, condição econômica e renda familiar), antropométricas (peso e altura) para classificação do estado nutricional segundo o Índice de Massa Corporal - IMC, questões de saúde (presença de doenças e autopercepção de saúde), hábitos de estilo de vida (uso de tabaco, consumo de álcool, estado nutricional, atividade física, controle de estresse e quantidade de horas de sono por dia ) e utilização de serviços de saúde.

\section{Resultados e Discussão}

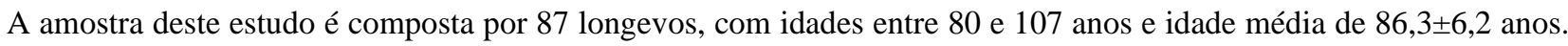
A maioria de participantes deste estudo foi do sexo feminino, viúvas e de baixa ou nenhuma escolaridade (Tabela 1) tendência comum em estudos com idosos longevos, a exemplo dos estudo de Dias et al. (2019), Steffens, Molinari e Dias (2019), Mota (2017), Grden, Weise, Reche, Borges e Cabral (2015) e Leite et al. (2010), tal qual encontrado neste trabalho, o que corrobora com Oliveira, Barbosa, Oliveira, Fernandes e Fernandes (2017), ao destacar o fenômeno da feminização do envelhecimento, devido ao processo de envelhecimento mais ativo e saudável relacionado aos indivíduos do sexo feminino, também atrelado aos cuidados com a saúde e menor exposição a situações de risco ocupacionais e de estilo de vida levados ao longo de toda a vida. 
Tabela 1 - Caracterização Sociodemográfica de longevos do município de Quixabeira-BA, Brasil, 2020.

\begin{tabular}{|c|c|c|c|c|c|}
\hline VARIÁVEL & $\mathbf{N}^{\mathbf{o}}$ & $\%$ & VARIÁVEL & $\mathbf{N}^{\mathbf{0}}$ & $\%$ \\
\hline SEXO & & & FILHOS & & \\
\hline Masculino & 41 & 47,1 & Não possui & 0 & 0,0 \\
\hline Feminino & 46 & 52,9 & De 1 a 5 filhos & 8 & 22,9 \\
\hline IDADE & & & De 6 a 10 filhos & 17 & 48,6 \\
\hline 80-89 anos & 65 & 74,7 & De 11 a 15 filhos & 8 & 22,9 \\
\hline 90-99 anos & 19 & 21,8 & $\geq 16$ filhos & 2 & 5,7 \\
\hline$\geq 100$ anos & 3 & 3,4 & ARRANJO DOMICILIAR & & \\
\hline RAÇA & & & Sozinho & 34 & 39,1 \\
\hline Branco & 14 & 16,1 & Conjugue & 14 & 16,1 \\
\hline Preto/Negro & 24 & 27,6 & Filho/s & 14 & 16,1 \\
\hline Pardo/Moreno & 47 & 54,0 & Neto/s & 4 & 4,6 \\
\hline Amarelo & 2 & 2,3 & Cônjuge e filho/s & 8 & 9,2 \\
\hline DENOMINAÇÃO RELIGIOSA & & & Cônjuge e neto/s & 5 & 5,7 \\
\hline Adventista do Sétimo Dia & 17 & 19,5 & Cônjuge, filho/s e neto/s & 1 & 1,1 \\
\hline Assembleia de Deus & 2 & 2,3 & Filho/s e neto/s & 7 & 8,0 \\
\hline Católica Apostólica Romana & 64 & 73,6 & CONDIÇÃO ECONOMICA & & \\
\hline Congregação Cristã no Brasil & 2 & 2,3 & Aposentado & 87 & 100,0 \\
\hline Não frequenta & 2 & 2,3 & RENDA FAMILIAR & & \\
\hline ESCOLARIDADE & & & <1 salário-mínimo & 4 & 4,6 \\
\hline Não estudou & 62 & 71,3 & 1 salário-mínimo & 18 & 20,7 \\
\hline Até 5 anos & 19 & 21,8 & De 1 a 3 salários-mínimos & 64 & 73,6 \\
\hline Entre 5 e 9 anos & 6 & 6,9 & $\geq 3$ salários-mínimos & 1 & 1,1 \\
\hline ESTADO CIVIL & & & & & \\
\hline Solteiro & 2 & 2,3 & & & \\
\hline Casado & 28 & 32,2 & & & \\
\hline Viúvo & 50 & 57,5 & & & \\
\hline Divorciado & 7 & 8,0 & & & \\
\hline
\end{tabular}

Fonte: Autores (2020).

Também alinhado a OMS ao destacar o gênero como um determinante transversal do envelhecimento ativo, fazendo inclusive menção ao papel tradicional da mulher como sendo a responsável pelo cuidadora da família, não tendo em muitos casos acesso ou eram forçadas a deixar de lado o trabalho remunerado em detrimento ao cuidado integral a família, enquanto que os homens apresentam maiores comportamentos de risco e de estar sujeitos a lesões ou morte devido à riscos ocupacionais e suicídio (WHO, 2005). Entretanto este perfil pode mudar em estudos futuros devido o novo arranjo familiar da então sociedade.

No que diz respeito ao arranjo domiciliar, a maioria dos idosos longevos deste estudo relataram residir sozinhos $39,1 \%$, valor semelhante ao encontrado no estudo de Grden et al. (2015), onde 46,4\% de longevos do município de Ponta Grossa no estado do Paraná residiam sozinhos. Cabendo aqui destacar a necessidade de atenção especial aos idosos longevos, os quais devido o processo de envelhecimento tendem a apresentar maior fragilidade e necessidade de cuidado e companhia, requerendo, portanto, atenção individualizada de forma que seja garantida a eles ambientes inclusivos, integradores e "capazes de favorecer o potencial do desenvolvimento humano”, maior autonomia e manutenção da capacidade funcional (Gonçalves, 2015).

Em nosso estudo a maioria dos participantes possuíam entre 6 a 10 filhos (42,5\%), corroborando com Leite et al. (2010), onde 56,47\% dos longevos avaliados também possuíam entre 6 a 10 filhos. Nesse contexto, segundo Oliveira, Silva \& Teixeira (2013), os idosos exercem um papel importante no sustento e manutenção da renda da rede familiar, isso porque, 
segundo os autores "os idosos estão se tornando atores sociais fundamentais para a manutenção de suas famílias [...] por meio de inúmeros fatores que influenciam as transferências financeiras que os idosos oferecem”, sendo, portanto, estes em muitos casos os principais apoiadores financeiros em diversas oportunidades.

Dessa forma cabe destacar, a importância da estabilidade econômica do idoso dado principalmente aos programas de previdência social existentes, garantindo a estes uma renda mínima mensal, o que foi possível observar neste estudo, onde os idosos longevos relataram possuir renda familiar de 1 a 3 salários mínimos 73,6\%, cuja fonte se deu proveniente da aposentadoria $100 \%$, corroborando com outros trabalhos realizados com longevos e descritos pela literatura (Grden, Weise, Reche, Borges \& Cabral, 2015; Mota, 2017; Dias et al., 2019; Steffens, Molinari \& Dias, 2019).

O risco e prevalência de DCNT tende a aumentar nos indivíduos em decorrência ao processo de envelhecimento motivado por suas alterações no que dizem respeito aos aspectos fisiológicos e biopsicossociais (Sardinha et al., 2020), nesse sentido a presença de DCNT em especial a HAS e DM como sendo as principais patologias encontradas nos idosos longevos de Quixabeira não nos surpreendeu (Tabela 2).

Tabela 2 - Presença de doenças em idosos longevos do município de Quixabeira-BA, Brasil, 2020.

\begin{tabular}{lcc}
\hline VARIÁVEL & $\mathbf{N}^{\mathbf{0}}$ & $\mathbf{\%}$ \\
\hline DESCRIÇÃO DA DOENÇA & & \\
CANCER & 1 & 1,1 \\
DM & 2 & 2,3 \\
CARDIOPATIA & 3 & 3,4 \\
LOMBALGIA & 5 & 5,7 \\
OUTROS & 9 & 10,3 \\
HAS & 15 & 17,2 \\
SEM DOENÇA & 15 & 17,2 \\
DOENÇA E ASSOCIAÇÕES & & \\
DM +1 & 4 & 4,6 \\
DM +2 & 3 & 3,4 \\
DM $\geq 3$ & 3 & 3,4 \\
HAS + 1 & 12 & 13,8 \\
HAS + 2 & 8 & 9,2 \\
HAS $\geq 3$ & 5 & 5,7 \\
LOMBALGIA +1 & 7 & 8,0 \\
LOMBALGIA +2 & 4 & 4,6 \\
LOMBALGIA $\geq 3$ & 5 & 5,7 \\
\hline
\end{tabular}

Fonte: Autores (2020).

A HAS foi a doença mais presente em longevos de um estudo realizado no estado do Rio Grande do Sul por Leite et al. (2010), cujo objetivo se deu em identificar o perfil e condições de saúde de idosos longevos, onde 65,88\% dos longevos avaliados possuíam a doença, enquanto a DM esteve presente em $9,41 \%$, sendo no referido estudo a quarta doença de maior prevalência, ainda, segundo os pesquisadores $16,47 \%$ dos longevos avaliados relataram não possuir problemas de saúde, valor semelhante ao encontrado neste estudo, onde 17,2\% dos longevos avaliados do município de Quixabeira relataram não possuir problemas de saúde, enquanto que a HAS também foi o problema de saúde de maior frequência entre os indivíduos pesquisados $46 \%$, seguido por lombalgias $24,1 \%$ e DM $13,8 \%$.

Ao avaliarem o comportamento de saúde e processo de envelhecimento de idosos entre 60 e 99 anos de idade, moradores de uma cidade no interior do estado de Minas Gerais, Scoralick-Lempke et al. (2018), constataram que problemas 
de visão atingiam a 79,2\% dos idosos 303 idosos avaliados, seguido de HAS 55,1\% e Dislipidemias 38,9\%, enquanto que o DM e doenças do coração estiveram presente em $20,8 \%$ e 19,8\% respectivamente, o que demonstra uma maior necessidade de prevenção e controle dos impactos ocasionados pela presença dessas doenças desde a mais tenra idade e de maneira especial dentro do grupo etário longevo.

A HAS, foi também a patologia mais presente entre os idosos longevos do estudo de Dias et al. (2019), correspondendo a $45,2 \%$, segundo os pesquisadores a HAS é tida como "um dos problemas de saúde atualmente de maior acometimento", o que reforça a ideia de que as DCNT, com destaque a HAS, podem ser consideradas as doenças mais frequentemente encontradas ao estudarmos populações longevas, conforme mencionado nos parágrafos anteriores e dado aos resultados encontrados durante a realização deste estudo, o que reforça a necessidade de atenção especial a essa população, aja visto que as doenças cardiovasculares e DCNT estão intimamente ligadas ao aumento da mortalidade entre idosos, segundo Cao et al. (2019).

Considerada por Borges et al. (2014), como um indicador relevante ao bem-estar e envolto a múltiplos fatores determinantes, a exemplo do sexo, faixa etária, suporte familiar, renda, capacidade funcional, condição de saúde, estilo de vida entre outros, a autopercepção de saúde entre os participantes desta pesquisa demonstrou resultado positivo, visto que 79,3\% dos idosos longevos pesquisados em Quixabeira apresentaram autopercepção boa ou ótima (Tabela 3), valor semelhante ao encontra por Bortoluzzi et al. (2018), ao avaliar a autopercepção de saúde de idosas praticantes de atividade física, onde 75,4\% das pesquisadas possuíam boa ou muito boa autopercepção de saúde, e também superiores ao encontrado no estudo de Borges et al. (2014), ao avaliar a autopercepção de saúde de idosos residentes em um município do interior do Rio Grande do Sul, a saber $61,3 \%$.

Tabela 3 - Autopercepção de Saúde e utilização de serviços de saúde por longevos do munícipio de Quixabeira-Ba, Brasil, 2020.

\begin{tabular}{lcc}
\hline VARIÁVEL & $\mathbf{N}^{\mathbf{0}}$ & $\mathbf{\%}$ \\
\hline AUTOPERCEPÇÃO DA SAÚDE & & \\
Péssima/ruim & 3 & 3,4 \\
Regular & 15 & 17,2 \\
Boa/ótima & 69 & 79,3 \\
UTILIZAÇÃO DE SERVIÇOS DE SAÚDE & & \\
Participa de grupo de saúde de idosos & 1 & 1,1 \\
Plano de saúde & 1 & 1,1 \\
Exclusivamente privado & 1 & 1,1 \\
Exclusivamente público & 40 & 46,0 \\
Público e privado & 45 & 51,7 \\
\hline
\end{tabular}

Fonte: Autores (2020).

A maioria dos longevos avaliados por este estudo relatou utilizar o serviço público e privado 51,7\% de saúde, seguido por aqueles que utilizam exclusivamente o serviço público de saúde $46 \%$, tal dado vem de encontro ao estudo realizado por Grden et al. (2014), onde os autores destacam as unidades básicas de saúde como sendo os locais mais procurados pelos idosos longevos correspondendo a 80,4\% dos pesquisados, ainda, segundo os autores, $28,6 \%$ dos longevos possuíam plano de saúde particular.

Cabe aqui destacar, baseado nestes achados a necessidade e importância de ações voltadas a promoção e recuperação da saúde, desenvolvidas principalmente pelos serviços de atenção primária quanto ao atendimento ao idosos, uma vez que esses serviços são considerados como porta de entrada aos serviços de saúde e necessitando, portanto, de maior capacitação, 
atenção e assistência prestadas por esses, voltadas a essa parcela da população de forma a garantir o atendimento adequado as necessidades e especificidades da população longeva (Grden et al., 2015).

Dessa forma, o estabelecimento de grupos de saúde e atenção ao idoso, tornam-se fundamentais no processo de universalização do sistema, por meio da garantia e prestação de serviços de conscientização, prevenção, detecção, recuperação e promoção da saúde integral do idoso, estando estes, intimamente alinhados aos determinantes do envelhecimento ativo propostos pela OMS (ILC Brasil, 2015).

Ao avaliarmos a participação dos idosos longevos de Quixabeira em grupos de saúde de idosos, a maioria dos entrevistados afirmam não fazer parte, correspondendo a 96,6\%, o que reforça a necessidade de maior incentivo, oferta de ambientes e grupos de idosos, atenção e assistência a esse grupo etário, voltada não apenas a prolongação da vida, mas, principalmente a manutenção da autonomia e independência dos indivíduos longevos por parte dos serviços de saúde e autoridades governamentais (Brito, Fernandes, Coqueiro, Jesus, \& Freitas, 2014).

A partir da análise dos dados deste estudo a abstinência ao cigarro/tabaco foi identificada em 87,4\% dos longevos (Tabela 4), valor similar ao encontrado por Cao et al. (2019), onde 86,2\% não eram fumantes. Valores semelhantes também foram encontrados no que diz respeito a abstinência ao álcool, onde 84,2\% dos longevos chineses avaliados por Cao et al. (2019) não faziam uso de bebidas alcoólicas, enquanto $85,1 \%$ dos longevos de Quixabeira também relataram não fazer uso de bebidas alcoólicas.

Tabela 4 - Hábitos de estilo de vida de idosos longevos do munícipio de Quixabeira-BA, Brasil, 2020

\begin{tabular}{lcc}
\hline VARIÁVEL & $\mathbf{N}^{\mathbf{0}}$ & $\mathbf{\%}$ \\
\hline USO DE TABACO & 11 & 12,6 \\
Fumante & 76 & 87,4 \\
Não fumante & & \\
USO DE ÁLCOOL & 74 & 85,1 \\
Abstinência/Não bebe & 2 & 2,3 \\
$\leq 4 x$ ao ano & 6 & 6,9 \\
$\leq 2 x$ ao mês & 1 & 1,1 \\
$\geq 4 x$ por semana & 4 & 4,6 \\
$\leq 3 x$ por semana & & \\
ESTADO NUTRICIONAL & 32 & 36,8 \\
Baixo peso & 33 & 37,9 \\
Eutrófico/adequado & 11 & 12,6 \\
Sobrepeso & 11 & 12,6 \\
Obesidade & & \\
ATIVIDADE FÍSICA & 34 & 39,1 \\
Pratica atividade física & 53 & 60,9 \\
Não pratica atividade física & & \\
CONTROLE DO ESTRESSE & 73 & 83,9 \\
Vive em ambiente não estressado & & \\
SONO & 80 & 92,0 \\
$\geq 8$ h de sono/diária (ideal) & 7 & 8,0 \\
<8h de sono/diária & & \\
\hline
\end{tabular}

Fonte: Autores (2020).

A presença do consumo de álcool e tabaco, segundo Barbosa, Pereira, Cruz e Leite (2018), é mais frequente em grupo de idosos mais jovens, tal fenômeno pode estar associado ao fato de que com o avançar da idade e aumento da fragilidade os idosos tendem a diminuir ou deixar o consumo dessas drogas. Para Scoralick-Lempke et al. (2018), o tabagismo e etilismo apresentam efeitos diretos na saúde, capacidade funcional e processo de envelhecimento, sendo, portanto, a utilização dessas 
substâncias um fator de risco para o desenvolvimento de DCNT em idosos.

Para Barbosa et al. (2018), "ações de saúde, políticas de prevenção e intervenção devem ser planejadas, utilizando uma abordagem integrada com o social, o psicológico e o biológico, visando diminuir o consumo abusivo do álcool e o tabagismo", são de fundamental importância, tendo em vista a diminuição dos danos ocasionados e associados ao consumo dessas drogas a saúde.

No estudo de base populacional com idosos do Brasil, realizado por Pereira, Spyrides e Andrade (2016), os pesquisadores observaram que 43,8\% dos idosos longevos do país apresentavam peso adequado, segundo o IMC, sendo que 26,3\% estavam com baixo peso, os autores destacam em seu estudo a relação direta existente entre o avanço da idade com o baixo peso, o que justifica o aumento de déficits nutricionais em idosos longevos, dessa forma, quanto maior a idade maior a prevalência do baixo peso para essa população.

Clementino e Goulart (2020), identificaram o peso adequado como predominante entre os idosos longevos avaliados, seguido pelos idosos com baixo peso, respectivamente 54,9\% e 14,7\%, tal sequência também se deu em nosso estudo, onde $37,9 \%$ os longevos avaliados apresentaram peso adequado e $36,8 \%$ baixo peso, a prevalência de peso adequado seguido por baixo peso também foi encontrada por Brito, Fernandes, Coqueiro, Jesus, \& Freitas (2014), em um estudo de base populacional em uma cidade do interior da Bahia, respectivamente $46,5 \%$ e $37,2 \%$.

A prevalência de baixo peso em idosos domiciliados está entre $1 \%$ a $15 \%$, entre $25 \%$ a $60 \%$ para idosos institucionalizados e de 35\% a 65\% em idosos hospitalizados (SBNPE, 2019). Embora comum entre idosos mais velhos o baixo peso e desnutrição é motivo de preocupação, em especial no que refere a idosos que vivem sozinhos na comunidade ou em instituições de longa permanência e internados em unidades hospitalares, dentre as possíveis causas para o baixo peso e desnutrição em idosos, destacam-se a diminuição do paladar, olfato, saciedade, déficits cognitivos, problemas relacionados a saúde bucal, diminuição ou incapacidades físicas, além de possíveis efeitos colaterais relacionados ao uso de medicamentos, doenças crônicas e depressão (ILC Brasil, 2015).

Segundo a OMS, "estimou-se que para cada um dólar investido em medidas para estimular a atividade física moderada houve uma economia de 3,2 dólares em despesas médicas", essa afirmação demonstra o quanto é importante e economicamente viável a prevenção de doenças e promoção da saúde através do estabelecimento e fortalecimento de hábitos saudáveis de estilo de vida, tal qual a prática de atividade física (WHO, 2005), dessa forma, é importante salientar que o envolvimento em atividades físicas pode prevenir o surgimento de doenças e declínio do estado funcional de idosos sadios ou doentes crônicos, o que é de extrema importância e necessidade ao público de idosos longevos.

Nesse contexto $39,1 \%$ dos longevos deste estudo relatam praticar algum tipo de atividade física, sendo que $24,1 \%$ exercitam-se sempre ou frequentemente durante as atividades habituais diárias, tais valores são semelhantes ao encontrado por Brito et al. (2014), onde $26,1 \%$ dos idosos pesquisados foram considerados fisicamente ativos, demonstrando, portanto, a necessidade de maior incentivo e promoção de vida ativa a essa população, a fim de promover a diminuição do surgimento de doenças, aja visto que segundo os resultados deste estudo, a atividade física exerce papel significativo e de fundamental importância para a maior ausência de doenças entre os idosos longevos avaliados.

A prática de atividade física está também associada a uma melhor saúde mental e maior contato social, além da redução do risco de quedas e maior autonomia, sendo necessário para tanto, a garantia de espaços e áreas seguras, tal qual a orientação e supervisão profissional objetivando a orientação e participação gradativa nas atividades e programas de atividade e reabilitação física, especialmente os indivíduos com algum comprometimento (WHO, 2005), tais ações podem vir inclusive a contribuir com a efetivação e fortalecimento de grupos dos grupos de saúde de idosos.

Visto que o ambiente pode contribuir para os níveis de estresse em idosos (Hernández, Phillips \& Siegel, 2016), o fato da maioria destes idosos $(83,9 \%)$ morarem em ambientes não estressantes indica um certo grau de controle e atenção quanto 
aos níveis de estresse, controle esse que segundo Souza, Porto, Souza e Silva (2016), pode ser um dos fatores a contribuir para a maior longevidade em idosos longevos.

A National Sleep Foundation (Hirshkowitz et al., 2015) recomenda entre 7 e 8 horas de sono para adultos mais velhos, o que corrobora com o ILC Brasil (2015), ao recomendar ao menos 8 horas de sono, destacando inclusive que "dormir regularmente duas horas a menos do que as oito horas de sono recomendadas aumenta o risco de obesidade, diabetes e doenças cardiovasculares", destacando a importância do repouso diário entre os idosos longevos, contribuindo para o envelhecimento saudável, nesse contexto os idosos longevos avaliados no município de Quixabeira demonstram em sua ampla maioria satisfação e cumprimento mínimo das horas de sono recomendadas, onde 94,3\% dos idosos desse estudo relatam estar satisfeito com o sono e $92 \%$ afirmam dormir igual a 8 horas de sono ou mais por noite.

\section{Conclusão}

Pode-se concluir que os idosos longevos desse estudo, maioria do sexo feminino, não alfabetizados e com renda familiar entre 1 e 3 salários-mínimos, possuem estado nutricional adequado, vivem em ambientes não estressantes, não fumam, não bebem e dormem pelo tempo mínimo recomendado para a idade, a prática de atividade física por esses idosos foi baixa e a HAS foi a doenças de maior prevalência. Estes indivíduos utilizam mais frequentemente os serviços públicos de saúde e tem autopercepção positiva quanto a saúde.

Estes achados, podem contribuir para a melhor compreensão do perfil de longevos residentes de pequenas cidades com baixo IDH, quanto aos aspectos sociodemográficos, de saúde e hábitos de estilo de vida, de forma que ações voltadas a promoção da saúde e hábitos de vida saudáveis sejam fortalecidos e implementados levando em consideração este público.

\section{Agradecimentos}

Agradecemos ao Centro Universitário Adventista de São Paulo (UNASP) pelo apoio e incentivo ao desenvolvimento deste trabalho, a Prefeitura Municipal de Quixabeira-BA pelo apoio logístico para realização da coleta de dados, aos voluntários que colaboraram para coleta de dados e de maneira especial aos idosos participantes que gentilmente colaboraram para realização deste trabalho.

\section{Referências}

Barbosa, M. B., Pereira, C. V., Cruz, D. T., \& Leite, I. C. G. (2018) Prevalência e fatores associados ao consumo de álcool e de tabaco em idosos não institucionalizados. Revista Brasileira de Geriatria e Gerontologia, 21(2), 125-135.

Bortoluzzi, E. C., Pancotte, J., Doring, M., Graeff, D. B., Alves, A. S., Portella, M. R., Scortegagna, H. M., \& Dalmolin, B. M. (2018). Autopercepção de saúde de idosas praticantes de atividade física e fatores associados. Estudos Interdisciplinares sobre o envelhecimento, 23(2), 119-231.

Borges, A. M., Santos, G., Kummer, J. A., Fior, L., Molin, V. D., \& Wibelinger, L. M. (2014) Autopercepção de saúde em idosos residentes em um município do interior do Rio Grande do Sul. Revista Brasileira de Geriatria e Gerontologia, 17(1), 79-86.

Brasil. Ministério da Saúde. Secretaria de Atenção à Saúde. Departamento de Atenção Básica. (2006). Envelhecimento e saúde da pessoa idosa. Brasília: Ministério da Saúde.

Brito, T. A., Fernandes, M. H., Coqueiro, R. S., Jesus, C. S., \& Freitas, R. (2014). Capacidade funcional e fatores associados em idosos longevos residentes em comunidade: estudo populacional no nordeste do Brasil. Fisioterapia e Pesquisa, 21(4), 308-313.

Buettner, D. \& Skemp, S. (2016). Blue Zones: Lessons from the World`s Longest Lived. American Journal of Lifestyle Medicine., 10(5), 318-321.

Cao, Z., Wang, R., Cheng, Y, Yang, H., Li, S., Sun, L., Xu, W., \& Wang, Y. (2019). Adherence to a healthy lifestyle counteracts the negative effects of risk factors on all-cause mortality in the oldest-old. AGING, 11(18), 7605-7619.

Clementino, M. D. \& Goulart, R. M. M. (2019). Imagem corporal, estado nutricional e qualidade de vida em idosos longevos. Revista Brasileira de Geriatria e Gerontologia, 22(6), e.190181. 
Dewes, J. O. (2013). Amostragem em Bola de Neve e Respondent-Driven Sampling: uma descrição dos métodos. (Monografia Bacharel em Estatística). Departamento de Estatística do Instituto de Matemática da Universidade Federal do Rio Grande do Sul, Porto Alegre, RS, Brasil. https://lume.ufrgs.br/bitstream/handle/10183/93246/000915046.pdf?sequence=1\&isAllowed=y

Dias, V. F., Araújo, L. S. L. R., Cândido, A. S. C., Lopes, A. O. S., Pinheiro, L. M. G., \& Reis, L. A. (2019). Dados sociodemográficos, condições de saúde e sinais de violência contra idosos longevos. Revista de Saúde Coletiva da UEFS, 9, 186-192.

Ferreira, L. K., Meireles, J. F. F., \& Ferreira, M. E. C. (2018). Avaliação do estilo e qualidade de vida em idosos: uma revisão de literatura. Revista Brasileira de Geriatria e Gerontologia, 21(5), 639-651.

Gonçalves, C. D. (2015). Envelhecimento Bem-Sucedido, Envelhecimento Produtivo e Envelhecimento Ativo: Reflexões. Estudos Interdisciplinares sobre o Envelhecimento, 20(2), 645-657.

Govindaraju, D., Atzmon, G., \& Barzilai, N. (2015). Genetics, Lifestyle and longevity: Lessons from centenarians. Applied \& Translational Genomics, 4, 2332 .

Grden, C. R. B., Weise, T., Reche, P. M., Borges, P. K. O., \& Cabral, L. P. A. (2015). Características sociodemográficas e de acesso de longevos aos serviços de saúde. Ciência, Cuidado e Saúde, 14(4),1505-1512.

Hernández, D., Phillips, D., \& Siegel, E. L. (2016). Exploring the Housing and Household Energy Pathways to Stress: A Mixed Methods Study. International Journal of Environmental Research and Public Health, 13(919), 1-13.

Hirshkowitz, M., Whiton, K., Albert, S. M., Alessi, C., Bruni, O., DonCarlos, L., Ware, J. C. (2015). National Sleep Fundation's updated sleep duration recommendations: final report. Sleep Health Jornal, 1(4), 233-243.

IBGE - Instituto Brasileiro de Geografia e Estatística. (2015). Mudança demográfica no Brasil no início do século XXI: subsídios para as projeções da população. Rio de Janeiro: IBGE.

IBGE - Instituto Brasileiro de Geografia e Estatística. (2010). Sinopse dos Resultados do Censo 2010: Distribuição da população por sexo, segundo os grupos de idade - Quixabeira (Ba). https://censo2010.ibge.gov.br/sinopse/webservice/default.php?cod1=29\&cod2=292593\&cod3=29\&frm=piramide

ILC Brasil - Centro Internacional de Longevidade Brasil. (2015). Envelhecimento Ativo: Um Marco Político em Resposta à Revolução da Longevidade. Rio Janeiro: ILC Brasil. http://longevidade.ind.br/wp-content/uploads/2017/03/Envelhecimento-Ativo-Um-Marco-Pol-tico-ILC-Brasil_web.pdf

Leite, M. T., Hildebrandt, L. M., Gonçalves, L. H. T., Falcade, B. L., Biasuz, S., Heisler, E. V., \& Ghedini, Q. P. (2010). Caracterização e condições de saúde de idosos ais idosos residentes em um município do norte do Rio Grande do Sul. Revista Brasileira de Ciências do Envelhecimento Humano, 7(1), 71-79.

Lima, S., Reis, Z., \& Souza, A. (2005). QUIXABEIRA: Uma Visão Geográfica da História. Jacobina: UNEB.

Lopes, M. A., Krug, R. R., Bonetti, A., \& Mazo, G. Z. (2014). Envelhecimento na Percepção das Pessoas Longevas Ativas e Inativas Fisicamente. Estudos Interdisciplinares sobre o Envelhecimento, 19(1), 141-153.

Minayou, M. C. S. \& Firmo, J. O. A. (2019). Longevidade: bônus ou ônus? Ciência \& Saúde Coletiva, 24(1), 1.

Mota, J. P. (2017). Características sociodemográficas, fragilidade e sarcopenia em idosos longevos (Dissertação de Mestrado). Escola de Saúde e Medicina da Universidade Católica de Brasília, Brasília, DF, Brasil. https://bdtd.ucb.br:8443/jspui/handle/tede/2184

Oliveira, M. B., Silva, N. M., \& Teixeira, K. M. D. (2013). Transferências financeiras: os idosos como suporte econômico familiar. Anais da Jornada de Ensino, Pesquisa e Extensão, Recife, PE, Brasil. 13. http://www.eventosufrpe.com.br/2013/cd/resumos/r0978-1.pdf

Oliveira, S. M., Barbosa, K. T. F., Oliveira, F. M. R. L., Fernandes, W. A. A. B., \& Fernandes, M. G. M. (2017). Determinantes sociales, económicos y ambientales de envejecimiento activo em ancianos atendidos en un centro de atención integral a la salud. Cultura de los Cuidados, 21(49), 155-165.

Pereira, I. F. S., Spyrides, M. H. C., \& Andrade, L. M. B. (2016). Estado nutricional de idosos no Brasil: uma abordagem multinível. Cadernos de Saúde Pública, 32(5), e00178814.

Pignolo, R. J. (2019). Exceptional Human Longevity. Mayo Clinic Proceedings, 94(1), 110-124.

PNUD - Programa das Nações Unidas para o Desenvolvimento. (2013). Atlas do Desenvolvimento Humano no Brasil: Quixabeira, BA. www.atlasbrasil.org.br/2013/pt/perfil_m/quixabeira_ba

Sardinha, M. T. M. U., Sá, S. P. C., Ferreira, J. B. S., Lindolpho, M. C., Domingos, A. M. \& Melo, V. L. (2020). Qualidade de vida de idosos com doenças crônicas e suas representações sociais. Research, Society and Development, 9(9), e30996470. Doi: http://dx.doi.org/10.33448/rsd-v9i9.6470

SBNPE - Sociedade Brasileira de Nutrição Parenteral e Enteral. (2019). Diretriz BRASPEN de terapia nutricional no envelhecimento. BRASPEN Journal, 34(3), 2-58.

Scoralick-Lempke, N. N., Nascimento, E., Ribeiro, B. C. S., Moreira, C., Oliveira, M. E. L., Souza, P. C., \& Teixeira, T. J. (2018). Comportamentos de saúde e envelhecimento saudável: um estudo com idosos da comunidade. Revista Família, Ciclos de Vida e Saúde ao Contexto Social, 6(4), 775-784.

Souza, M. A. H., Porto, E. F., Souza, E. L., \& Silva, K. I. (2016). Perfil de estilo de Vida de longevos. Revista Brasileira de Geriatria e Gerontologia, 19(5), 819-826.

Steffens, T., Molinari, T., \& Dias, C. P. (2019). Relação entre estado cognitivo e variáveis sociodemográficas e funcionais em idosos longevos: estudo observacional no município de Porto Alegre/RS. Estudos Interdisciplinares em Envelhecimento, 24, 61-74. 
Research, Society and Development, v. 10, n. 3, e33810312839, 2021

(CC BY 4.0) | ISSN 2525-3409 | DOI: http://dx.doi.org/10.33448/rsd-v10i3.12839

UNDP - United Nations Development Programme. (2015). Human Development Report 2015: Work for Human Development. UNDP.

WHO - World Health Organization. (2005). Envelhecimento ativo: uma política de saúde. OPAS. 